\title{
Quality control before chromosome detection in peripheral blood
}

\author{
Yanwei Guo ${ }^{1}$, Zhigao Long*2 \\ ${ }^{1}$ Department of Clinical Laboratory, Baogang Hospital of Inner Mongolia, Baotao, Inner Mongolia, China \\ ${ }^{2}$ State Key Laboratory of Medical Genetics, College of Life Science, Central South University/Hunan Jiahui genetics \\ Hospital/Prenatal Diagnosis Center, Xiangya Hospital, Central South University, Changsha, China
}

Received: October 11, 2017

DOI: $10.14725 /$ dcc.v4n4p21
Accepted: November 16, $2017 \quad$ Online Published: December 10, 2017

URL: http://dx.doi.org/10.14725/dcc.v4n4p21

\begin{abstract}
The chromosome detection in peripheral blood of 1,863 patients in our hospital from January 2011 to June 2016 were analyzed. The quality control and precautions before chromosome detection in peripheral blood were reported as follows.
\end{abstract}

Key Words: Chromosome detection, Quality control

The accreditation criteria for quality and capability of medical laboratories issued by the National Accreditation Committee of conformity assessment of China in November 22, 2013 (CNAS-CL02) divide the whole process into three stages. That is, before the test, during the test and after the test. ${ }^{[1]}$ The influence factors on the quality of inspection are different at different stages. Cytogenetic laboratory should take full account of the factors affecting chromosome detection and strictly control it. ${ }^{[2]}$ It is found that $80 \%$ of quality problems that affect the results of the test are caused by the pre-test factors. ${ }^{[3]}$ Therefore, quality control before inspection is the key to ensure the quality of chromosome detection. Before the test, the quality control should include patient history collection, patient preparation, specimen collection and specimen transport storage. In this paper, the results of chromosome detection in peripheral blood of 1,863 patients in our hospital from January 2011 to June 2016 were analyzed. At the same time, combined with related literatures, the practical experience, quality control and precautions before chromosome detection in peripheral blood were analyzed as follows.

\section{Specimen test results analysis}

Based on the test results of peripheral blood chromosome in 1,863 patients, we preliminarily found that the pre-test factors which affected the results of chromosome detection included patient history collection, patient preparation, specimen collection, specimen transportation and storage, culture medium and consumables quality. The main influence factors and the impact on the results were shown in Table 1.

\footnotetext{
*Correspondence: Zhigao Long; E-mail: 115678696@qq.com; Address: State Key Laboratory of Medical Genetics, College of Life Science, Central South University/Hunan Jiahui genetics Hospital/Prenatal Diagnosis Center, Xiangya Hospital, Central South University, Changsha, China.
} 
Table 1: Analysis of influential factors before major inspection

\begin{tabular}{|c|c|c|c|c|}
\hline Patients \&specimens & $\mathbf{n}$ & Cell culture & Split and banding & Processing method \\
\hline Usage of antibiotics & 21 & 2 cases of failure & Bad split, hard and unclear banding & Blood re-collection \\
\hline After angiography & 7 & $\begin{array}{l}2 \text { cases of failure, } 6 \text { cases of } \\
\text { success }\end{array}$ & Bad split, hard and unclear banding & Blood re-collection \\
\hline Bacterial contamination & 14 & Few cells & $\begin{array}{l}\text { Small cell accounts for a big part, less } \\
\text { split, hard banding }\end{array}$ & Blood re-collection \\
\hline lipoidemia and chylemia & 11 & Few cells & $\begin{array}{l}\text { Small cell accounts for a big part, less } \\
\text { split, hard banding }\end{array}$ & Blood re-collection \\
\hline Hemolysis & 4 & Few cells & Less split, unclear banding & Blood re-collection \\
\hline
\end{tabular}

\section{Discussion}

Comprehensive collection of patient history is essential to ensure the quality of chromosomal detection results, especially in patients with chromosomal disease. When the patient's history information indicates that there is a factor that affects the karyotype analysis, the examiner will increase the analysis count, thus finding a lower proportion of chimerism. If the collection of medical history is incomplete and lack of relevant information, the examiner may ignore the abnormal karyotype and cause the failure to report. In our clinical work, there was 1 case of infertility. 20 split phases were counted and 5 karyotypes were analyzed. The results showed that there were no other abnormalities except the Y chromosome was suspected of qh+. The C band detection showed that 1 split phase was $47, \mathrm{XYY}$, after increasing the number of split phase to 100,7 split phases were found to be 47, XYY. The final determination of the patient's karyotype was 46, XY [93]/47, XYY [7]. The results of the return visit showed that the patient had asthenospermia accompanied by the syndrome of 47 , XYY. It may lead to a missed diagnosis if the $\mathrm{C}$ band detection is not carried out just because the relevant information is not collected during medical history collection before the test. In order to ensure the integrity of patient's history information, clinicians should collect history information, make tabular information about diseases that need to be done in laboratory, and fill them out in the process of history collection, so as to prevent omissions. ${ }^{[4]}$

An appointment to collect blood is usually required for chromosomal detection in peripheral blood. We should simply ask about the situation of the patient, such as the medical condition at the time of appointment. If the patient has conducted other tests, patients should be advised to carry the test results when collecting the specimen so as to facilitate the collection of information. Antibacterial drugs, contrast agents and other drugs can cause failure of lymphocyte culture, less lymphocyte harvesting, chromosome splitting and chromosome banding and so on. Therefore, in the collection of medical history, we must understand the patient's drug use in the near future. If the patient is taking antibacterials or contrast agents in the near future, the patient should be told to collect the peripheral blood 1 week later. In the appoint- ment, the patient should take at least 8 hours of empty stomach before collecting the peripheral blood, so as to avoid the difficulty of cell culture caused by lipoidemia and chylemia.

The collection of peripheral blood specimens should be used in vacuum extraction. Anticoagulants are recommended to use heparin sodium or heparin lithium anticoagulant tubes. After blood collection, the mixture should be gently inverted several times. The anticoagulant should be fully exposed to the blood. ${ }^{[5]}$ Chromosome detection of peripheral blood specimens generally needs to be cultured for $68-72 \mathrm{~h}$, and the medium is very suitable for bacterial growth. Therefore, in the collection of specimens, we must pay attention to aseptic technique and specified disinfection to avoid contamination of specimens leading to cell culture failure. ${ }^{[6]}$

During the transportation of the peripheral blood specimens, severe sloshing and heat preservation should be averted in order to avoid the hemolysis of the specimens. ${ }^{[7]}$ When the specimen is collected from the laboratory, it should be informed of the notices in the course of transportation. Especially when the temperature difference between indoor and outdoor is greater, we should pay more attention to the heat preservation and transportation of the specimen. Once the specimen is hemolytic, it will result in fewer lymphocytes, fewer splits, difficult banding and unclear stripes. The preservation time of the peripheral blood specimen should not exceed $8 \mathrm{~h}$ at room temperature. The specimens that cannot be inoculated on the same day and the specimens after the inoculation can be preserved at $4^{\circ} \mathrm{C}$. The results of inoculation are acceptable when the specimens are preserved within 7 days at $4^{\circ} \mathrm{C}$, but the preservation could not last more than 3 days. ${ }^{[8]}$

Peripheral blood chromosomes can be detected using the prepared medium or laboratory self-culture medium. Glutamine is an essential amino acid for the synthesis of nucleic acids and proteins, therefore, a 1640 medium containing glutamine should be selected for the laboratory selfmatching medium. Due to the instability of glutamine, the medium should be added with the same amount of glutamine as the original medium after being stored for more than 1 week at $4^{\circ} \mathrm{C}$. Both the prepared medium and the selfmatched medium should be preserved at $-20^{\circ} \mathrm{C}$ and avoid repeated freezing and thawing. In order to verify the quality of 
the culture medium, the original culture medium should be compared with the original culture medium for each batch. The medium that does not meet the requirements of quality should not be used.

Experimental supplies such as vacuum blood collection tubes, syringes, centrifuge tubes, one pipette and other unqualified quality can also lead to failure of chromosome preparation experiments such as cell culture failure, cell harvest failure or banding difficulties and so on. Therefore, after the replacement of experimental materials, the quality of the new consumables should be tested before they could be used. Consumables quality testing can also be used in parallel experiments and it is best to detect only one kind of consumable material in each experiment so as to find out the problem.

The detection of chromosomes in peripheral blood, as one of the methods of cytogenetic detection, has some limitations due to most of them are manual detection. There are many steps from specimen collection to report experiment, among

\section{References}

[1] China National Accreditation Committee for conformity assessment. CNAS-CL02 Standards for accreditation of medical laboratory quality and ability. Beijing: China National Accreditation Committee for conformity assessment; 2012.

[2] Zhang L, Wang W, Wang ZG. Management of pre- and post-test stages. Chin Hosp Manag. 2015; 35(8): 34-36.

[3] Cong YL. Quality management and Countermeasures before clinical analysis. Chin J Lab Med. 2004; 27(8): 483.

[4] Yan H, Guo XX, Bai Y. Effect of clinical infiltration and information feedback path on pre test quality control. Chin J Clinicians (Electronic Edition). 2016; 10(1): 155-158.

[5] Wu YY, Cao LL, Deeng ZL. The influence of blood specimen collection on the result of biochemical test. Jilin Med J. 2014; 35(8): them human factors are the influencing factors. The failure of any link may cause the failure of the experiment, even the result of error or misdiagnosis of. ${ }^{[9]}$ There are many factors affecting the test results before inspection, which involve all aspects, and the quality control before inspection is often ignored. To solve the quality problem of peripheral blood chromosome detection before testing, we should firstly improve the quality assurance work at the early stage of analysis, and raise awareness of the importance of specimen quality, especially the patient's information collection and patient preparation. ${ }^{[10]}$

Pre-test quality control of chromosome testing is an important prerequisite and basis for ensuring the accuracy of test results. The cell genetic laboratory should attach importance to the pre-test factors affecting chromosome detection and strictly control it.

\section{Conflicts of Interest Disclosure}

The authors have no conflicts of interest related to this article.

1581-1582.

[6] Tang WJ, Zuo Y, Xu Z. Chromosomal analysis of peripheral blood and quality control in 862 cases of genetic counseling. Chinese Journal of Birth Health \& Heredity. 2015; 23(2): 25-26.

[7] Huang KB, Yang XM, Ye M. Analysis of pre analysis quality control of laboratory medicine. Int J Lab Med. 2015; 36(3): 428-429.

[8] Qiu HG, Lin H, Hou XQ. Factors influencing the preparation of chromosome $\mathrm{G}$ banding in human peripheral blood. Chin Prac Med. 2011; 6(27): 225-227.

[9] Long ZG, Xing EH, Wang YZ. Prenatal diagnostic techniques and specifications for cytogenetics. Chin J Prac Gynecol Obstetr. 2015; 31(9): 811-814.

[10] Shang H, Wang YS, Shen ZY. National operating procedures for clinical examination. $4^{\text {th }}$ edition. Beijing: People's Medical Publishing House; 2015. 1003-1012 p. 